\title{
Atomic Scale Analysis of Chemical Intermixing in MBE-grown GaSb/InAs Superlattices Based on Z-contrast Imaging
}

\author{
Honggyu Kim ${ }^{1,2}$, Yifei Meng ${ }^{1,2}$, Jean-Luc Rouviére ${ }^{3}$, and Jian-Min Zuo ${ }^{1,2}$ \\ 1. Dept. of Materials Science and Engineering, University of Illinois, Urbana, Illinois 61801, USA \\ 2. Seitz Materials Research Laboratory, University of Illinois, Urbana, Illinois 61801, USA \\ 3. CEA/INAC/SP2M/LEMMA, 19 rue des Martyrs, 38054 Grenoble, France
}

Type-II superlattices (T2SLs) consisted of GaSb and InAs have great potential for applications in laser and photodetectors operating in mid-wavelength to lo ng-wavelength infrared (IR) regime [1]. In comparison to the conventional $\mathrm{HgCdTe}$ (HCT) based detectors, T2SLs offer several advantages such as band-gap tunability, reduced tunnelling current, and compatibility with III-V semiconductor production [2]. Additionally, theory predicts a longer Auger lifetime in T2SLs and significant detector performance advantage compared to HCT detectors with the same energy ga p [3]. Until rece ntly, the detector performance of fabricated T2SLs, however, was inferior compared to HCT detectors and does not meet the theoretical prediction [4]. Interfacial intermixing and roughness are among the factors suggested that could contribute to the degraded IR detector performance. The state-of-the-art MBE enables the growth of strain balanced GaSb/InAs T2SLs without forming misfit dislocations. The chemical intermixing at the interfaces is, however, unavoidable due to the growth kinetics [5]. Investigation of in terfaces in $\mathrm{GaSb} / \mathrm{InAs}$ T2SLs is essential to obtain chemical information across the interfaces and further optimize growth conditions. Scanning transmission electron microscopy (STEM) and template matching image processing technique are employed for this study.

We have developed the image processing technique based on template matching for quantitative interface analysis. Firstly, the template images are created from a Z-con trast image obtained with a STEM using a high angle annular dark field (HAADF) detector. Imaged along the [110] zone axis, the cation-anion pairs of zinc-blend structures like GaSb and InAs appears as so-called dumbbell structures. These dumbbell structures for GaSb and InAs are distinguishable in a Z-contrast image because of large difference in atomic numbers. The templates for GaSb and InAs are created by spatially averaging over many repeated patterns in each layer to reduce the noise in an as-recorded Z-contrast image. The Zcontrast image is, then, matched with two tem plates. The amplitude values, which contain quantitative contribution of each template to the recorded Z-contrast image, are extracted by linear decomposition. Fig. 1(a) presents a high magnification Z-contrast image of T2SL recorded with the electron energy of $300 \mathrm{kV}$. A T2SL was grown on GaSb substrate. The thicknesses of GaSb and InAs are $2.2 \mathrm{~nm}$ and 4.4 $\mathrm{nm}$, respectively to target a specific IR wavelength. The GaSb and InAs tem plates, obtained by spatial averaging, are displayed in Fig. 1(b) and (c) with reduced noise level. Using amplitude values obtained with template matching technique, the c hemical distribution of GaSb and In As is quantitatively displayed as amplitude maps in Fig. 2 (a) and (b). At interface regions, both GaSb and InAs peaks are detected, which suggests chemical intermixing of GaSb and InAs. The lin e profile along growth direction as shown in Fig. 2 (c) indicates that InAs-on-GaSb interfaces are more extended than GaSb-onInAs interfaces. Also, the amplitude value of GaSb in T2SL is le ss than that of GaSb substrate. This result presents the GaSb in T2SL is chemically mixed with InAs components.

Using template matching technique with atomic resolution Z-contrast images, we have demonstrated atomic scale analysis of chemical intermixing in MBE-grown GaSb/InAs T2SLs. The comparison 
between GaSb/InAs T2SLs with different growth schemes will be also discussed.

\section{References:}

[1] D. L. Smith et al, Journal of Applied Physics 62 (1987), 2545.

[2] D. R. Rhiger, Journal of Electronic Materials 40 (2011), 1815.

[3] C. H. Grein, Appl. Phys. Lett. 61 (1992), 2905.

[4] W. E. Tennant, Journal of Electronic Materials 39 (7) (2010), 1030.

[5] R. Magri, Phys. Rev. B 65 (16) (2002), 165302.

[6] This work is supported by U.S. Army Research Office (grant number Army W911NF-10-1-0524 and monitored by Dr. William Clark) through the MURI program. Electron microscopy performed at CEA, Grenoble, France, was supported by Nanoscience foundation, France. Lastly, we thank Prof. Shun Lien Chuang's group for a fruitful discussion about device physics.

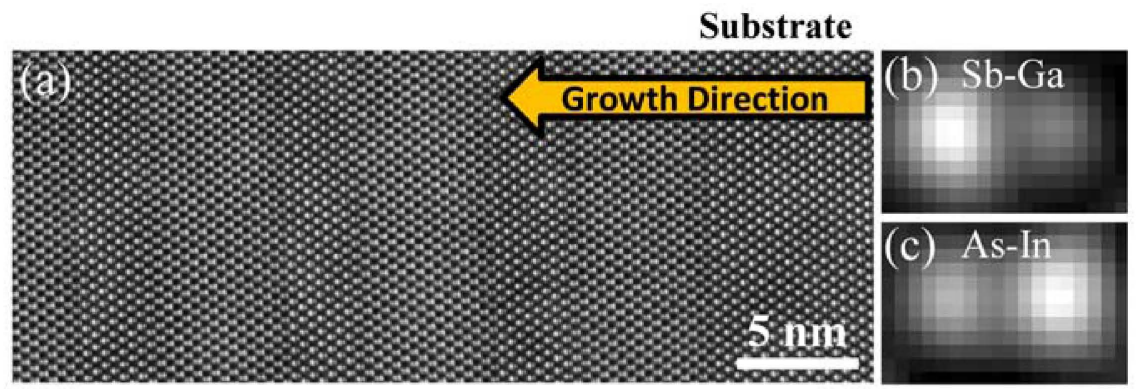

Figure 1. (a) Z-contrast image obtained with the el ectron energy of $300 \mathrm{kV}$. (b) GaSb and (c) InAs templates.

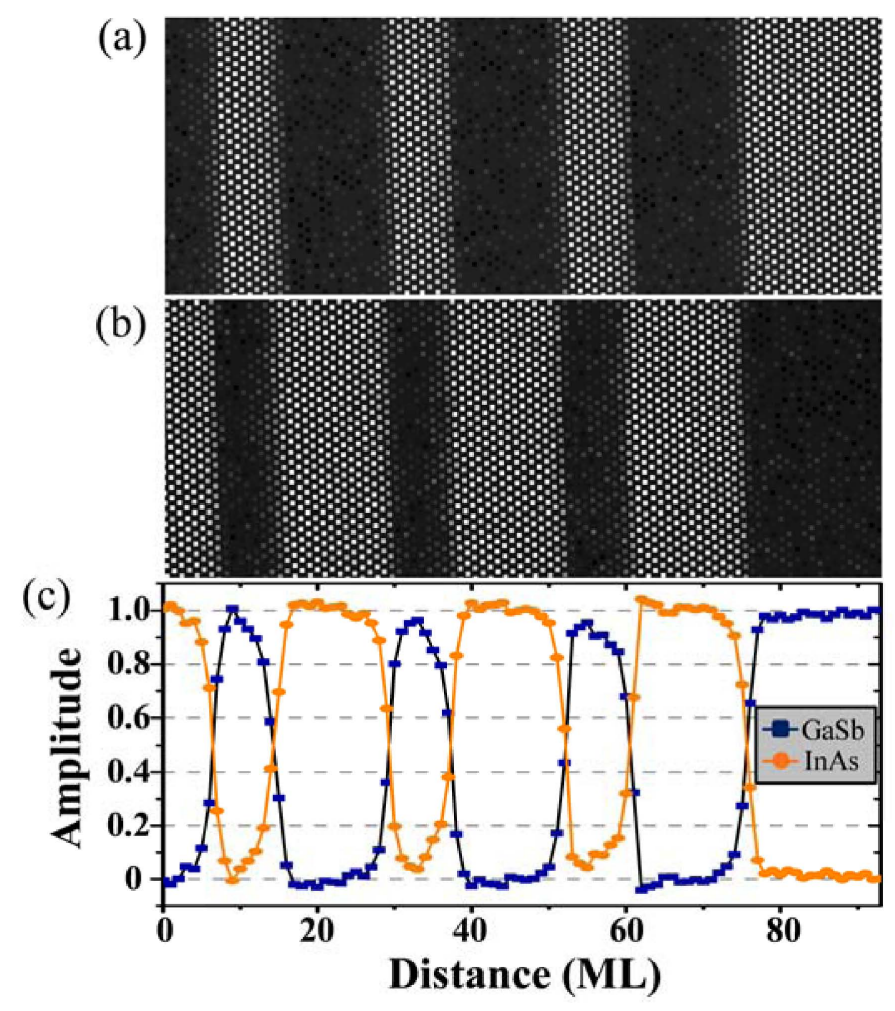

Figure 2. (a) GaSb and (b) InAs amplitude maps. (c) Line profiles of GaSb and InAs obtained from amplitude maps. 